\title{
Kardiovaskuläre Erkrankungen
}

\section{iPS-abgeleitete Kardiomyozyten als therapeutische Hoffnungsträger}

\author{
NINA GRILL ${ }^{1}$, LISA FELLNER ${ }^{1,2}$, MARCEL TISCH ${ }^{2}$, FRANK EDENHOFER ${ }^{1,2}$ \\ 1 VASCAGE GMBH, INNSBRUCK, ÖSTERREICH \\ 2 INSTITUT FÜR MOLEKULARBIOLOGIE, ABTEILUNG FÜR GENOMIK, STAMMZELL- \\ BIOLOGIE UND REGENERATIVE MEDIZIN UND CENTER FOR MOLECULAR BIOSCIENCES \\ INNSBRUCK (CMBI), UNIVERSITÄT INNSBRUCK, ÖSTERREICH
}

\section{After ischemic injury in adolescence, the human heart has only limited capacity to regenerate. The loss of cardiomyocytes is typically replaced by fibrotic scar tissue. The resulting scars reduce myocardial contractility and function. To date, the gold standard for end-stage heart disease remains a heart transplant, which is not a realistic option due to donor heart shortage. The regenerative potential of reprogrammed stem cells has the potential for a long-expected breakthrough in development of efficient therapeutic interventions.}

DOI: $10.1007 / \mathrm{s} 12268-021-1609-1$

(c) Die Autoren 2021

Das menschliche Herz weist nach ischämischen Verletzungen in der Adoleszenz nur eine limitierte Regenerationskapazität auf. Als Konsequenz gleicht der Körper den Verlust von Kardiomyozyten typischerweise mit der Entstehung von fibrotischem Narbengewebe aus. Das Myokard vernarbt und die Kontraktilität des Herzmuskels wird vermindert. Bis heute bleibt der Goldstandard für Patient/inn/ en mit Herzerkrankungen im Endstadium die Herztransplantation, welche jedoch durch den
Mangel an Spenderherzen für die meisten Patient/inn/en keine realistische Option darstellt. Ein grundlegender Durchbruch in der Entwicklung neuer und effizienter therapeutischer Methoden ist daher dringend notwendig.

Die rasante Entwicklung der Stammzellforschung in den letzten Jahren liefert zwei neue therapeutische Optionen: i) in vivoRegeneration des Myokards durch Mobilisierung und Anregung vorhandener Vorläuferzellen zur Zellteilung und Differenzierung und ii) Transplantation von in vitro generierten Zellen. Verschiedene Zellquellen wurden als potenzielle Kandidaten für die Myokardreparatur identifiziert, u. a. Skelettmyoblasten [1], Knochenmarkstammzellen [2], residente Herzvorläuferzellen [3], vaskuläre Stammzellen in der Adventitia [4] und pluripotente Zellen, wie embryonale Stammzellen (ES) oder induzierte pluripotente Stammzellen (iPS-Zellen) [5].

\section{Das Potenzial der iPS-Zellen}

Im Jahr 2007 gelang der Forschergruppe des japanischen Stammzellforschers Shinya Yamanaka erstmalig die Reprogrammierung menschlicher Fibroblasten zu einem pluripotenten Status [6]. Die Faszination dieser induzierten pluripotenten Stammzellen liegt in der Möglichkeit, Zellen aller drei Keimblätter zu generieren. So lassen sie sich nicht nur in Zellen des Gehirns und anderer innerer Organe differenzieren, sondern auch in Zellen des Herzens, wie z. B. Kardiomyozyten. Eine weitere Besonderheit: Sie können aus Fibroblasten- oder Blutzellen von Patient/inn/en hergestellt werden und spiegeln somit die genetischen Gegebenheiten der jeweiligen Person wider (Abb. 1).

Für die Reprogrammierung der Fibroblasten werden die Yamanaka-Faktoren (Oct3/4, Sox2, Klf4 und c-Myc) verwendet, die durch

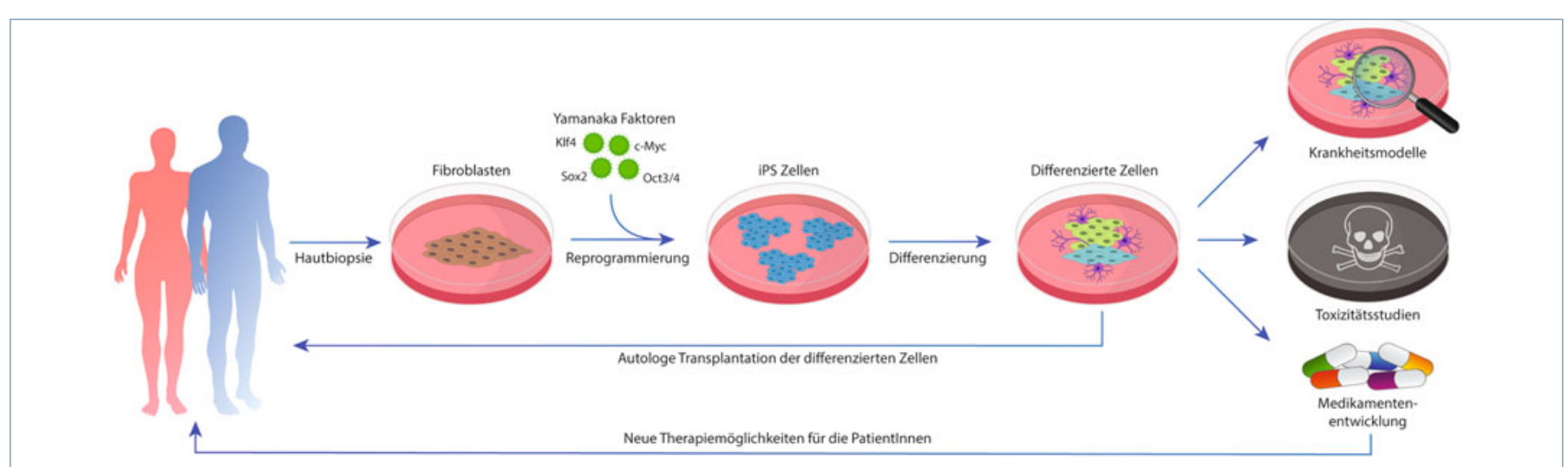

A Abb. 1: Herstellung und Verwendung von hiPS-Zellen in der Biomedizin. Somatische Zellen werden durch eine Hautbiopsie gewonnen und durch die Yamanaka-Faktoren (Kfl4, c-Myc, Sox2 und Oct3/4) zu hiPS-Zellen reprogrammiert. Die Differenzierung zu einem spezialisierten Zelltyp (u.a. Kardiomyozyten) ermöglicht Anwendungen der autologen Transplantation in der Zellersatz-Therapie, Entwicklung von Krankheitsmodellen, sowie Toxizitätsstudien und Medikamentenentwicklungen. 
verschiedene, integrierende und nicht integrierende Methoden in die Zellen eingebracht werden können. Die iPS-ZellTechnologie ermöglicht nicht nur personalisierte therapeutische Interventionen, wie myokardiale Patches, sondern ist auch ein neues, zielführendes Werkzeug bei der Analyse pathophysiologischer Konsequenzen genetischer Prädispositionen. Durch die Verwendung von patienteneigenen Zellen kann nicht nur die Wirkung bekannter Medikamente auf die spezielle, genetisch bedingte Zellkonstitution getestet werden, auch so genannte Wirkstoffdatenbanken können auf eventuelle Wirksamkeit untersucht werden. Außerdem erhofft man sich hieraus eine Etablierung neuer Therapiemöglichkeiten, wie die autologe Zellersatztherapie mit in vitro differenzierten Zellen (Abb. 1).

\section{iPS-Zellen als vielversprechende Quelle für kardiale Therapieoptionen}

Ein Beispiel für die Anwendung patientenspezifischer iPS-Zellen und daraus abgeleiteter spezialisierter Zellen findet man in der Kardiologie: Die Herstellung von Herzmuskelzellen aus iPS-Zellen. Diese Kardiomyozyten mit ihren prominenten kontraktilen Filamenten stellen zwar nur einen von vielen Zelltypen des Herzens dar, doch spielen sie bei Herzinfarkten und Kardiomyopathien eine zentrale Rolle. So wird beispielsweise bei Myokardinfarkten der Verlust von Kardiomyozyten mit fibrotischem Narbengewebe ausgeglichen, das allerdings keine Kontraktilität aufweist.

Um diesen Verlust von Myokard auszugleichen, könnten im Rahmen einer zellbasierten Regenerationsstrategie Kardiomyozyten aus iPS-Zellen in vitro hergestellt und transplantiert werden. Für die Herstellung von Kardiomyozyten aus iPS-Zellen gibt es verschiedene Möglichkeiten, die hervorragend in einem Review von C. L. Mummery et al. zusammengefasst werden (Abb. 2A, [7]). Grundsätzlich lassen sich die Herstellungsmethoden in 2D- und 3D-Ansätze unterscheiden (u. a. Embryoidkörperchen, induktive Ko-Kultur), von denen sich jedoch die Monolayer-basierte Differenzierung als robustes Protokoll mit relativ einfacher Handhabung, als Standard etabliert hat.

Generell durchlaufen humane iPS-Zellen während der Differenzierung zu Kardiomyozyten drei Stadien, die durch biphasi- sche Regulierung des WNT-Signalwegs geprägt sind (Abb. 2B):

(1) die Induktion des Mesoderms, das durch die Expression von Brachyury (T) gekennzeichnet ist,

(2) die Spezifizierung des kardiovaskulären Mesoderms mit Expression des kinase insert domain receptor (KDR) und platelet-derived growth factor receptor alpha (PDGFRA) und

(3) die Reifung der spezifischen Vorläuferzellen zu funktionalen Kardiomyozyten [8].

Nach Erlangung der Kontraktilität reifen die jungen Kardiomyozyten und stellen dabei die Proliferation überwiegend ein. Einige Studien zeigen zwar, dass es im gesunden Säugetierherzen eine gewisse, wenn auch äußerst geringe, Grundproliferation der Kardiomyozyten gibt [9], doch führt diese kaum vorhandene Proliferationskapazität zu einer Herausforderung in der Herstellung und Expansion dieser Zellen im industriellen Maßstab. J. W. Buikema et al. [10] konnten kürzlich einen neuen Ansatz zur Expansion mittels reversibler Wiederherstellung der Proliferation vorstellen. Unter Zugabe von CHIR99021, einem WNT-Agonisten, und Reduzierung der Kontaktinhibition wurde eine 100-250fache Expansion berichtet.

Durch die Identitätsveränderung im Verlauf der kardialen Differenzierung exprimieren die Kardiomyozyten nun zelllinienspezifische Marker, wie das Oberflächenprotein signal-regulatory protein alpha (SIRPA) und die kardialen Muskelmarker kardiales Troponin T (cTNT) und actinin alpha 2 (ACTN2), die zur Charakterisierung herangezogen werden können (Abb. 2C). Eine Aufreinigung der Kardiomyozyten-Zellpopulation kann beispielsweise durch Ersatz von Glucose mit Laktat im Nährmedium erreicht werden, da Kardiomyozyten im Gegensatz zu anderen Zellen Laktat verstoffwechseln können [8].

Eine zentrale Herausforderung ist die Frage, wie in vitro differenzierte, aufgereinigte und expandierte Kardiomyozyten-Kulturen idealerweise transplantiert werden, um ein geschädigtes Herz zu regenerieren. Da ein Einbringen von Kardiomyozyten an die betroffenen Stellen des Herzens ohne Matrix zu einem großen Verlust von Zellen nach der Transplantation führt und die Herzregeneration dadurch nicht ausreichend gewährleistet werden kann, scheinen 3D- oder 4D-Ansätze, wie eine Cardiac-Patch-Thera-

\section{Hier steht eine Anzeige.}

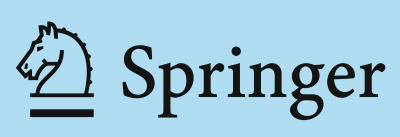




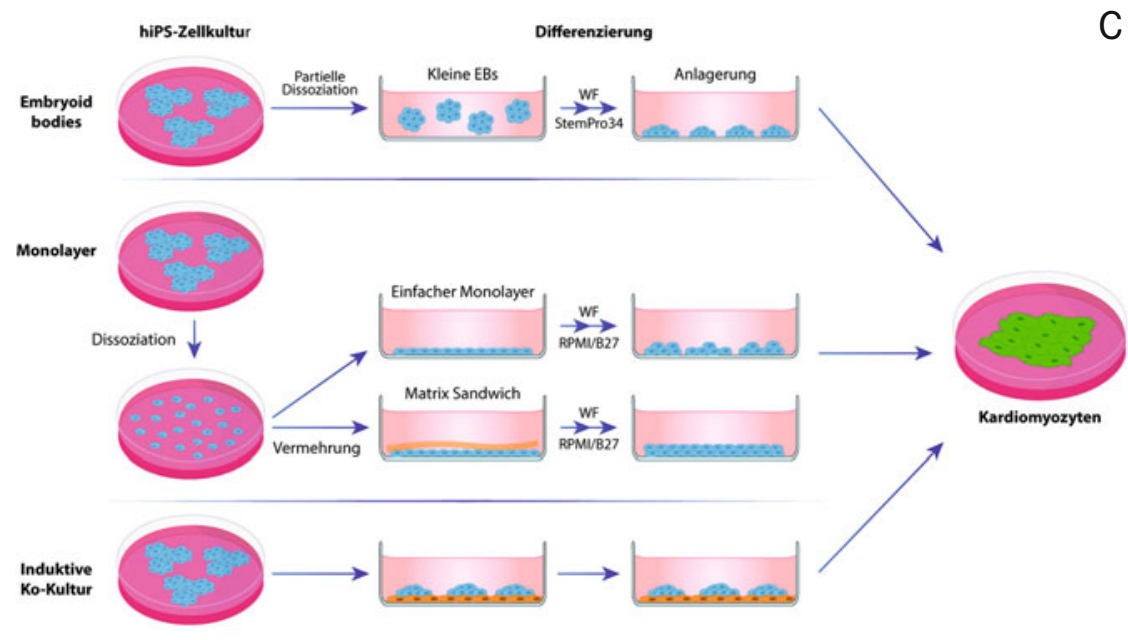

B

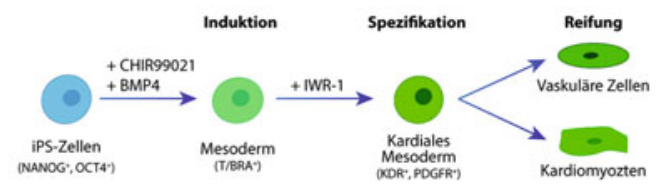

C
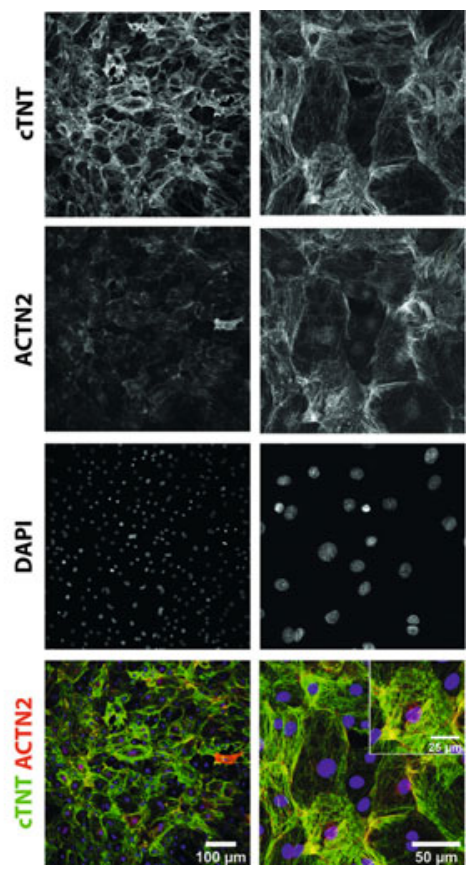

A Abb. 2: Von iPS-Zellen zu Kardiomyozyten: Methoden und Phasen der kardialen Differenzierung. A, Etablierte Methoden der kardialen Differenzierung: (1) Embryoidkörperchen (embryoid bodies, EBs) sind unkontrolliert differenzierte, aggregierte hiPS-Zellkolonien, die in Suspension wachsen. Durch Zugabe von Wachstumsfaktoren (WF) adhärieren die EBs und es reifen Kardiomyozyten daraus hervor. (2) hiPS-Zellen werden dissoziiert, zu einem Monolayer oder Matrix Sandwich vermehrt und über WF differenziert. (3) Induktive Ko-Kultur: hiPS-Zellen werden mit Induktor-Zellen ko-kultiviert und zu Kardiomyozyten differenziert. B, Zeitliche Abfolge bei der Kardiomyozyten Differenzierung: CHIR99021 (WNT-Agonist) und Bone Morphogenetic Protein 4 (BMP4) induzieren das Mesoderm, die kardiovaskuläre Spezifikation erfolgt durch IWR-1 (Inhibition des WNT-Signalweg; gekennzeichnet durch Kinase Insert Domain Receptor (KDR) und Platlet Derived Growth Factor Alpha (PDGFRA)), Reifung der Vorläuferzellen zu Kardiomyozyten. C, Immunzytochemische Färbung früher Kardiomyozyten: kardiales Troponin T in grün (cTNT) und actinin alpha 2 in rot (ACTN2); zusätzlich wurde eine Zellkernfärbung mit 4',6-Diamidin-2-phenylindol (DAPI, blau) durchgeführt.

pie, vielversprechend. Dabei werden Ko-Kulturen von Fibroblasten, mesenchymalen Stammzellen und Kardiomyozyten in ein biologisches, auf Kollagen basierendes, vaskularisiertes Gerüst eingebracht. Nach erfolgreicher Vaskularisation und Validierung kann der CardiacPatch transplantiert werden - bisherige Experimente am Tier zeigen hier bereits Erfolge [11, 12]. Allerdings bedarf es weiterer Untersuchungen und Experimente, um diese Methode erfolgreich beim Menschen einsetzen zu können.

\section{Danksagung}

Wir danken dem Fonds zur Förderung der wissenschaftlichen Forschung (FWF) und dem COMET-Zentrum VASCage, welches durch die österreichischen Bundesministerien BMK, BMDW sowie durch die Länder Tirol, Salzburg und Wien gefördert wird. Das Programm COMET wird durch die Forschungsförderungsgesellschaft FFG abgewickelt.

\section{Literatur}

[1] Menasché P (2007) Skeletal myoblasts as a therapeutic agent. Prog Cardiovasc Dis 50: 7-17

[2] Liao R, Pfister O, Jain M, Mouquet F (2007) The bone marrow-cardiac axis of myocardial regeneration. Prog Cardiovasc Dis 50: $18-30$

[3] Wu SM, Chien KR, Mummery C (2008) Origins and fates of cardiovascular progenitor cells. Cell 132: 537-543
[4] Mekala SR, Wörsdörfer P, Bauer J et al. (2018) Generation of cardiomyocytes from vascular adventitia-resident stem cells. Circ Res 123: 686-699

[5] Murry CE, Keller G (2008) Differentiation of embryonic stem cells to clinically relevant populations: lessons from embryonic development. Cell 132: 661-680

[6] Takahashi K, Yamanaka S (2006) Induction of pluripotent stem cells from mouse embryonic and adult fibroblast cultures by defined factors. Cell 26: 663-676

[7] Mummery CL, Zhang J, Ng ES et al. (2012) Differentiation of human ES and iPS cells to cardiomyocytes: A Methods Overview. Circ Res 111: 344-358

[8] Kadari A, Mekala S, Wagner N et al. (2015) Robust generation of cardiomyocytes from human iPS cells requires precise modulation of BMP and WNT signaling. Stem Cell Rev Rep 11: 560-569

[9] Senyo SE, Steinhauser ML, Pizzimenti CL et al. (2013) Mammalian heart renewal by pre-existing cardiomyocytes. Nature 493: 433-436

[10] Buikema JW, Lee S, Goodyer WR et al. (2020) Wnt activation and reduced cell-cell contact synergistically induce massive expansion of functional human iPSC-derived cardiomyocytes. Cell Stem Cell 27: 50-63

[11] Schürlein S, Al Hijailan R, Weigel T et al. (2017) Generation of a human cardiac patch based on a reendotheli alized biological scaffold (BioVaSc). Adv Biosyst 1: 1600005 [12] Cui H, Liu C, Esworthy T et al. (2020) 4D physiologically adaptable cardiac patch: A 4-month in vivo study for the treatment of myocardial infarction. Sci Adv 6: 26

Funding note: Open access funding provided by University of Innsbruck. Open Access: Dieser Artikel wird unter der Creative Commons Namensnennung 4.0 International Lizenz veröffentlicht, welche die Nutzung, Vervielfältigung, Bearbeitung, Verbreitung und Wiedergabe in jeglichem Medium und erlaubt, sofern Sie den/die ursprünglichen Autor(en) und die Quelle
ordnungsgemäß nennen, einen Link zur Creative Commons Lizenz beifügen und angeben, ob Änderungen vorgenommen wurden. Die in diesem Artikel angeben, ob Anderungen vorgenommen wurden. Die in diesem Artikel
enthaltenen Bilder und sonstiges Drittmaterial unterliegen ebenfalls der genannten Creative Commons Lizenz, sofern sich aus der Abbildungslegende nichts anderes ergibt. Sofern das betreffende Material nicht unter der genannten Creative Commons Lizenz steht und die betreffende Handlung nicht nach gesetzlichen Vorschriften erlaubt ist, ist für die oben aufgeführten Weiterverwendungen des Materials die Einwilligung des jeweiligen Rechteinhabers einzuholen. Weitere Details zur Lizenz entnehmen Sie bitte der
Korrespondenzadresse:

Univ.-Prof. Dr. Frank Edenhofer

Abteilung für Genomik, Stammzellbiologie \&

Regenerative Medizin

Institut für Molekularbiologie \& $\mathrm{CMBI}$

Leopold-Franzens-Universität Innsbruck

Technikerstraße 25

A-6020 Innsbruck

Frank.Edenhofer@uibk.ac.at

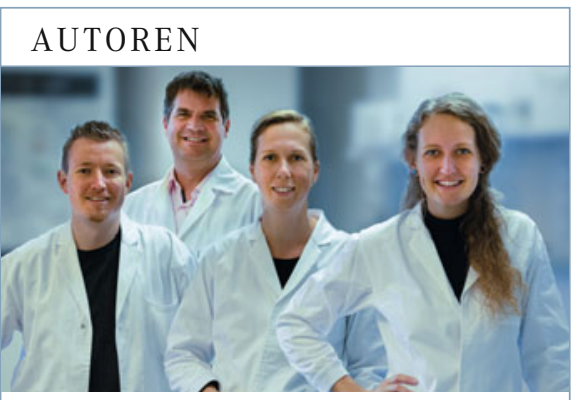

Marcel Tisch, Frank Edenhofer, Lisa Fellner und Nina Grill (v. I. n. r.).

\section{Arbeitsgruppe}

Die Forscher/innen der Abteilung Genomik, Stammzellbiologie \& Regenerative Medizin unter der Leitung von Prof. Dr. F. Edenhofer entwickeln und verwenden zelluläre Reprogrammierungsmethoden, um menschliche Krankheiten auf molekularer und zellulärer Ebene eingehend zu analysieren und um den Weg für neuartige therapeutische Ansätze zu ebnen. Der Fokus liegt hierbei auf degenerativen Erkrankungen des Nerven- und Herz-Kreislauf-Systems. 